\title{
Optimal slit orientation for long multi-object spectroscopic exposures ${ }^{\star}$
}

\author{
G. P. Szokoly
}

Max-Planck-Institut für extraterrestrische Physik, Giessenbachstraße, 85748 Garching, Germany

e-mail: szgyula@mpe.mpg.de

Received 17 December 2004 / Accepted 20 June 2005

\section{ABSTRACT}

Historically, long-slit spectroscopic observations were carried out using the parallactic angle for the slit orientation if slit loss was an important consideration (either to maximize the signal-to-noise or to do spectrophotometry). This requires periodic realignment of the slit position angle as the parallactic angle changes. This is not possible for multi-slit observations where one slit position angle must be chosen for the entire exposure. Common wisdom suggests using the parallactic angle at the meridian $(\mathrm{HA}=0)$. In this paper, I examine what the best strategy is for long, multi-slit exposures. I find that in extreme cases (very long exposure time) the best choice is to orient the slit perpendicular to the parallactic angle at the meridian. There are two effects to consider: the increasing dispersion with increasing airmass and the changing angle between the parallactic angle and the slit. In the case of traditional slit orientation, the two effects amplify each other, thus rendering a significant fraction of the observation useless. Using the perpendicular orientation, the two processes work against each other, thus most of the observation remains useful. I will use, as an example, our $8 \mathrm{~h}$ Lockman Hole observations using the Keck telescope, but generic methods are given to evaluate a particular observation. I also make the tools available to the community.

Key words. atmospheric effects - instrumentation: spectrographs - techniques: spectroscopic

\section{Introduction}

The introduction of multi-object spectrographs (MOS) in optical spectroscopy is quite often thought of as just a set of traditional long-slit spectrographs (LS) and some consequences are overlooked. The main difference between MOS and LS spectroscopy is the constraints imposed by the geometry of the instrument. While in LS spectroscopy, the slit orientation can be chosen arbitrarily, this is no longer the case in MOS spectroscopy: slit position angles cannot be changed during a set of observations.

As observers tend to concentrate on very faint objects (typical $R$-band magnitude of 23-24 with 8-10 m class telescopes), the integration times are longer and longer. Sometimes a whole night (8-10 h or more) is spent on a single mask. These observations require a rethinking of the optimal observation strategy.

Since many MOS instruments lack an atmospheric dispersion corrector (e.g. VIMOS on VLT, DEIMOS on Keck), atmospheric dispersion is a serious problem. Long observations (many hours) span a large range of zenith distance. Thus, a differential refraction of a few arc seconds is quite common.

As most observations require high signal to noise ratios or good spectral resolution, using sufficiently wide slits to compensate for this effect is not acceptable. Very wide slits have a

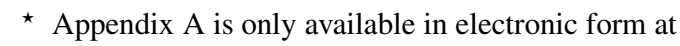
http://www. edpsciences.org devastating effect on background-limited exposures (since the sky background grows linearly with slit width, while the object signal grows much more slowly) and a wide slit also blurs the spectra.

For short exposures, one can observe "close to the parallactic angle", i.e. align the slit with the atmospheric dispersion direction. This way the photons from the object enter the slit, the dispersion only introduces an additional tilt in the resulting spectra, which is easy to correct for for most applications. If the goal of the observation is to extract spatial information, too, then extra care is required to correct for this effect.

In the case of longer observations, the direction of dispersion projected on the sky, i.e. the parallactic angle, varies with time. For single object observations (i.e. long slit spectroscopy), one can compensate by periodically realigning the slit. For MOS exposures, this is not possible. For masks, a single slit orientation must be chosen for the whole exposure.

In this paper, I examine how the effective slit loss can be estimated and I demonstrate the effect using our sample observation of the Lockman Hole using the Keck telescope. I also describe how to use our Web-based service to find the best strategy for a particular observation.

I will start with the current best determination of the atmospheric refraction. I also work out a simplified formula that is sufficient in many applications. I simulate different 
Table 1. Current and future $8 \mathrm{~m}$ class telescopes and MOS instruments.

\begin{tabular}{llcc}
\hline \hline Telescope & Instrument & Spectral range $(\AA)$ & ADC \\
\hline Keck & DEIMOS & $4100-11000$ & no \\
Keck & LRIS & $3100-10000$ & planned \\
Subaru & FOCAS & $3650-9000$ & yes \\
Gemini & GMOS & $3600-11000$ & yes \\
LBT & MODS & $3300-11000$ & yes \\
HET & LRS & $4150-9100$ & yes \\
SALT & PFIS & $3200-8500$ & yes \\
GTC & OSIRIS & $3650-10000$ & evaluating \\
VLT & FORS & $3300-11000$ & yes \\
VLT & VIMOS & $3300-11000$ & no \\
\hline
\end{tabular}

observational strategies and show the effect of atmospheric refraction on the efficiency of observations.

\section{Atmospheric dispersion and large telescopes}

The importance of atmospheric refraction in spectroscopic observations was emphasized by Filippenko (1982). The paper discusses the optimal strategy (and the effect of non-optimal strategies) for short long-slit spectroscopic observations. Even though the paper uses a formula to calculate the index of refraction that became obsolete, it is still the strategy to be followed for short integrations. The refined formula to calculate the refraction introduces only negligible changes. On the other hand, the paper does not discuss the optimal strategy for long integrations.

Cohen \& Cromer (1988) calculate the magnitude of differential refraction for the Keck and the Norris spectrographs for realistic observing scenarios. The paper determines the limits beyond which the atmospheric dispersion degrades the data, but does not discuss how to optimize observations that go beyond these limits.

Donnelly et al. (1989) discusses optimal observational strategies for fiber spectrographs for long exposures. Unfortunately these results cannot be directly applied to slit spectrographs: in many (but not all) projects, the slit orientation can be chosen arbitrarily, thus, there is an extra degree of freedom to minimize the effect of atmospheric refraction. This is not possible for fiber spectrographs, thus, this is not discussed in this paper.

In Table $1 \mathrm{I}$ review all current and known future $8 \mathrm{~m}$ telescopes and optical MOS spectrographs. There are only 2 instruments without an ADC in operation or in planning:

DEIMOS on Keck is heavily red optimized, thus, atmospheric dispersion is not a significant issue for many projects. The DEIMOS Slitmask design page (http : / / www .ucolick.org/ phillips / deimos_ref/ masks.html) provides preliminary tools to evaluate the effect of atmospheric dispersion on slit loss for short integrations. No guidelines are provided for long integrations.

The VIMOS manual (2005) discusses the effect of atmospheric dispersion in MOS mode. They arrive at the conclusion that the only generic way to minimize slit losses is to orient the slits North-South and observe within $\pm 2 \mathrm{~h}$ of the meridian.
Even though the detailed study of the effect of atmospheric dispersion on VIMOS (Cuby et al. 1998) makes no explicit statement about deviating from these constraints, a casual reading of the manual by an inexperienced observer may leave the impression that this slit orientation is the only valid strategy.

This conclusion is clearly valid for the sample observation used in the manual: observing between airmasses of 1.7 and 1.4 in the $\mathrm{UV} / \mathrm{blue}$. On the other hand, for some observing projects, e.g. limiting UV/blue spectroscopy to low airmass, the advantage of N-S orientation diminishes. Thus, an additional freedom (slit orientation) is available in some cases to maximize scientific return of the observations.

\section{Sample observation}

Throughout this paper, I will use a sample observation of the Lockman Hole $(\delta=+57: 35: 25.0$, J2000) using the Keck telescope (latitude of $+19: 46: 36$ ). I assume multislit spectroscopy with $1.0^{\prime \prime}$ slit width and $1.2^{\prime \prime}$ seeing. I assume an ambient temperature of 2.5 degrees $C$, ambient pressure of $61.5 \mathrm{kPa}$ (615 mbar) and a relative humidity of $40 \%$.

The observations consist of 1 night long integration on a mask, which for this field implies an hour angle range between $18 \mathrm{~h}$ and $3 \mathrm{~h}$ (the asymmetry is due to the mechanical constraints of the telescope), which covers an airmass range of 1.27 (hour angle of 0 ) to 3.5 (hour angle of $18 \mathrm{~h}$ ). I will concentrate on the DEIMOS multiobject spectrograph, which is red-optimized. Thus, I will concentrate on the $4500 \ldots 9500 \AA$ wavelength range.

In the calculation I assume that the seeing does not depend on the wavelength and I also assume that the alignment and guiding is done in the $R$-band (approximately $7000 \AA$ ).

\section{Atmospheric dispersion}

The most up-to-date atmospheric dispersion determination, the Ciddor formula (1996) is reviewed in the Appendix. The most important formulas are:

The differential refraction (as a function of wavelength, relative to the alignment/guiding effective wavelength, $\lambda_{0}$ ) in radians is:

$\Delta R(\lambda) \equiv R(\lambda)-R\left(\lambda_{0}\right)=\left(n(\lambda)-n_{0}\right) \tan z_{a}$.

In Fig. 1 I plot $\Delta R$ as a function of wavelength at different wavelengths for different airmasses.

The index of refraction, $n_{\mathrm{as}}$ of standard air is

$10^{8}\left(n_{\mathrm{as}}-1\right)=\frac{5792105 \mu \mathrm{m}^{-2}}{238.0185 \mu \mathrm{m}^{-2}-\sigma^{2}}+\frac{167917 \mu \mathrm{m}^{-2}}{57.362 \mu \mathrm{m}^{-2}-\sigma^{2}}$,

where $\sigma$ is the wave number (reciprocal of the vacuum wavelength) in inverse micrometers.

If we are only interested in differential refraction, we can write a simpler formula that is sufficiently accurate for many applications:

$\Delta R(\lambda) \approx \frac{p T_{0}}{p_{0} T}\left(n_{\mathrm{as}}(\lambda)-n_{\mathrm{as}}\left(\lambda_{0}\right)\right) \tan z_{\mathrm{a}}$. 


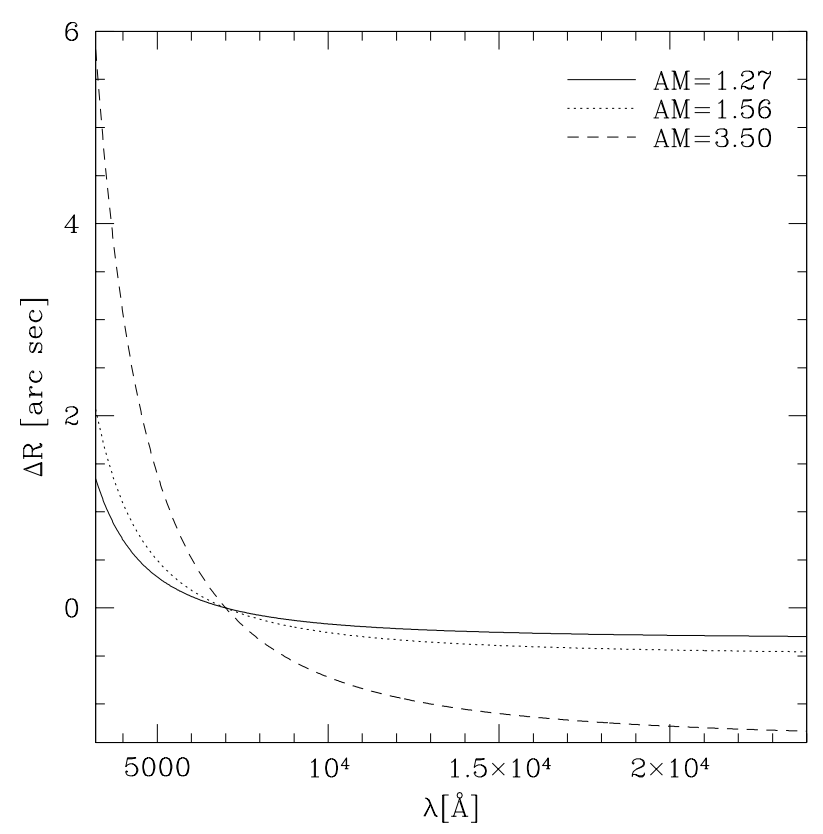

Fig. 1. Differential refraction as a function of wavelength at different airmasses: Solid line $-\mathrm{AM}=1.27(\mathrm{HA}=0)$, dotted line $-\mathrm{AM}=1.56$ $(\mathrm{HA}=3 \mathrm{~h})$, short dashed line $-\mathrm{AM}=3.50(\mathrm{HA}=18 \mathrm{~h})$. I used our sample observation of the Lockman Hole using Keck (see Sect. 1 for details).

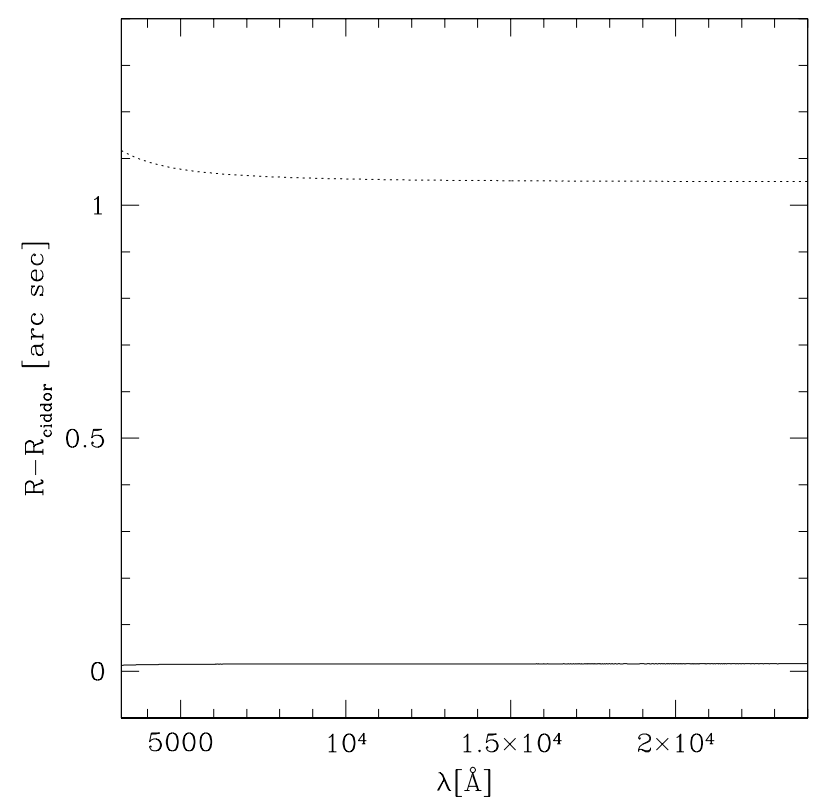

Fig. 2. The error introduced by our approximate formula (solid line) and the old Edlén formula (dotted line) in the atmospheric refraction assuming $\tan z_{\mathrm{a}}=1$.

The error introduced by this approximation (as well as the error introduced by the old Edlén formula) is shown in Fig. 2. As one can see, even the absolute refraction is well reproduced by this simpler formula, while the old Edlén formula is significantly different. For the calculation of the differential refraction, there is no practical difference between the formulas in the wavelength range considered.

\section{Slit loss}

Now that we have the atmospheric dispersion, we can also calculate the slit loss. I assume a point source that has a surface brightness profile of

$\mu(r)=\frac{1}{2 \pi \sigma^{2}} \mathrm{e}^{-r^{2} / 2 \sigma^{2}}$.

If we assume a slit of width $2 a$, that is sufficiently long, the fraction of light entering the slit from an object that is displaced by $x_{0}$ perpendicular to the slit (a displacement parallel to the slit does not affect the amount of light entering the slit) is

$I\left(x_{0}\right)=\frac{1}{\sigma \sqrt{2 \pi}} \int_{-a+x_{0}}^{a+x_{0}} \mathrm{e}^{-x^{2} / 2 \sigma^{2}} \mathrm{~d} x$.

The perpendicular displacement, $x_{0}$, depends on the differential refraction, $\Delta R$, and the angle between the slit and and parallactic angle. In Fig. 3 I consider two configurations, an East-West oriented slit (i.e. the slits are perpendicular to the parallactic angle at the meridian) and a North-South orientation (slits are parallel to the parallactic angle at the meridian).

As we can see, the N-S slit orientation results in a low slit loss at hour angle of 0 (minimal airmass) that does not depend on wavelength (as we are observing close to the parallactic angle). On the other hand, as we are moving away from the optimal configuration, the situation deteriorates rapidly. This is due to the fact that the atmospheric dispersion increases as the airmass increases and the slit orientation is moving away from the ideal, parallactic angle - both effects increase the slit loss.

In the alternative configuration, i.e. East-West slit orientation, the slit loss is never optimal. Even at low airmass, a significant fraction of the light is lost (e.g. slit loss is $42 \%$ at $5000 \AA$, instead of $33 \%$ ), but the slit loss does not deteriorate very quickly. This is due to the fact that as the airmass increases, the dispersion increases, but the slit is getting closer to the parallactic angle, thus the projected dispersion is not increasing very rapidly. In fact, in our particular configuration (Lockman Hole and Keck), the slit loss is actually smaller at high airmass $(42 \%, 38 \%$ and $39 \%$ at $5000 \AA$ at the airmass of $1.27,1.56$ and 3.50 , respectively).

To evaluate the overall effect of the slit orientation on the signal level achievable, we can also calculate the "average" slit loss of a long exposure. This is shown in Fig. 4.

In Fig. 5 I show the effect of sky position angle on average slit loss at $4500 \AA$, using my example observations and a southern field (Chandra Deep Field South). As we can see, for short exposures around meridian passage, the N-S orientation is optimal. As we go to longer integration times, the effect of slit orientation becomes smaller, while at extremely long integration times, the E-W orientation becomes ideal for the Lockman Hole field. For asymmetric cases (for example our actual observations between hour angles 18 and 3) the optimal slit orientation is neither E-W nor N-S.

In contrast, for the southern field, the optimal slit orientation remains North-South. This is due to the fact that for the CDFS field the airmass is never below 1.48 from Keck, thus, the differential refraction is comparable to the slit width even at meridian passing. 

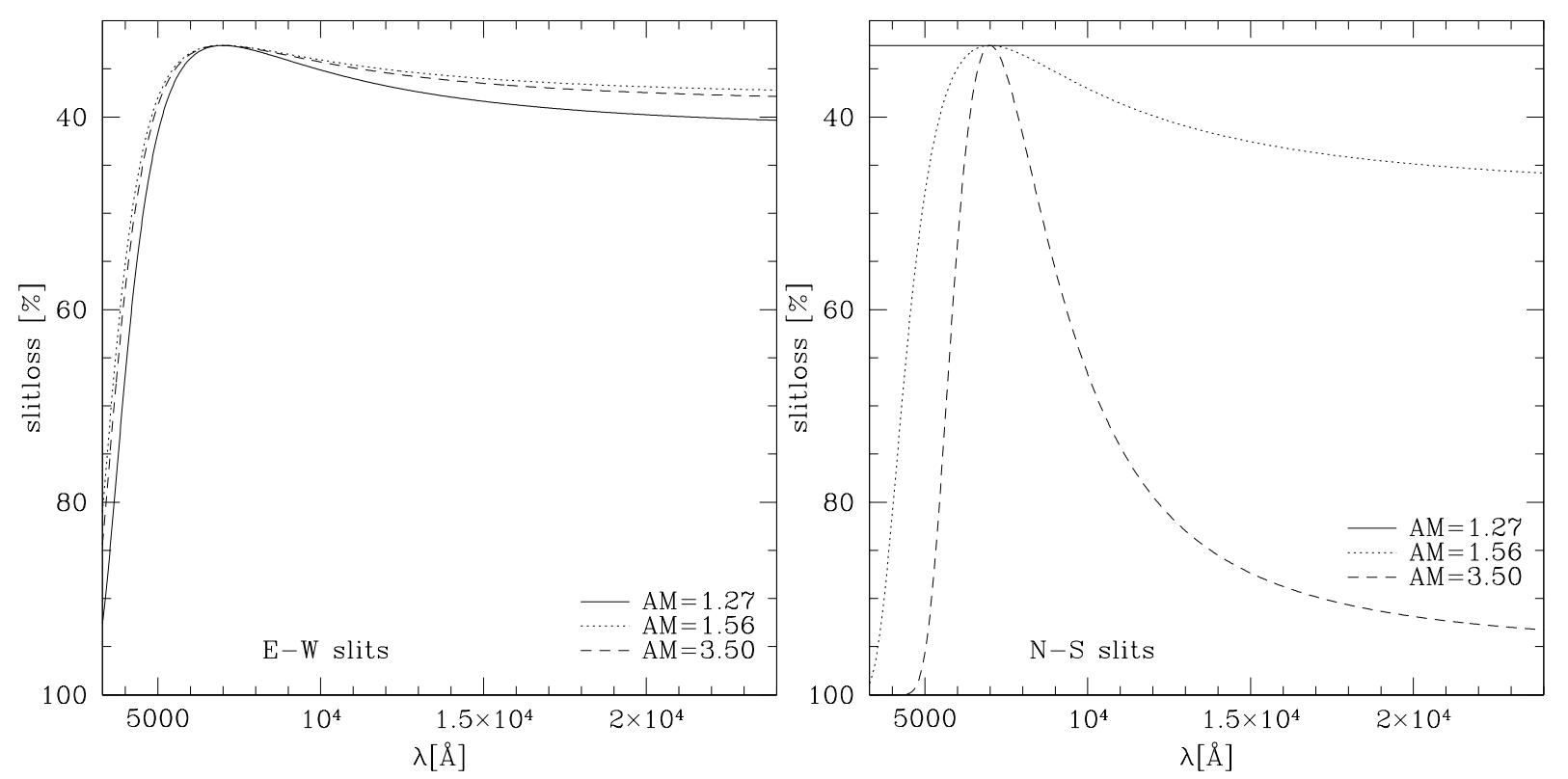

Fig. 3. Slit loss as a function of wavelength at different airmasses. I used our sample observation of the Lockman Hole using Keck (see Sect. 1 for details). Notice the different scales used on the plots.

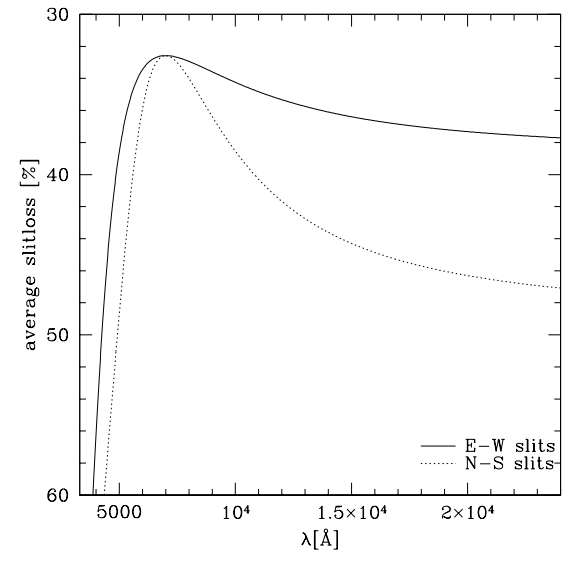

Fig. 4. Average slit loss at different slit orientations. Solid line indicates East-West slit orientation, dotted lines shows North-South orientation. I used our sample observation of the Lockman Hole using Keck (see Sect. 1 for details).

\section{Field differential refraction}

All results presented so far only hold for objects in the center of the field of view of the instrument. For wide-field spectrographs, an additional effect is important: the zenith distance is not constant across the field of view, thus the differential refraction is not constant, either. This is an achromatic effect, which we can calculate using Eq. (A.4):

$\delta R \approx(n-1) \frac{\mathrm{d} \tan z_{\mathrm{a}}}{d z_{\mathrm{a}}} \delta z_{\mathrm{a}}=(n-1) \sec ^{2} z_{\mathrm{a}} \delta z_{\mathrm{a}}$,

where $\delta R$ is the variation in the differential refraction across the field and $\delta z_{\mathrm{a}}$ is the field of view. As we have seen, $(n-1)$ is of the order of $3 \times 10^{-4}$, sec $z_{\mathrm{a}}$ is of the order of 1 , thus a 1000 arcsin field of view (nearly 17 arcmin) introduces a field differential refraction of the order of 0.3 arcsec. This effect makes it imperative to realign the masks periodically unless guiding is near the center of the field or the telescope compensates for off-axis guiding.

\section{Web interface}

I make our code used in our calculations available to the community in both source code and web application form at http://www . xray.mpe.mpg.de/ szgyula/slitloss/. The program uses the full Ciddor formula to evaluate the slit loss, but for comparison all three formulas are available to calculate the differential refraction.

\section{Conclusions}

I demonstrated that choosing the optimal slit orientation for multiobject spectroscopy, using long exposure times, requires care and should be evaluated individually for each project. For each field and expected duration, one has to find a balance.

I reviewed the most recent determination of atmospheric dispersion. For typical cases, I found that a simplified version of the most up-to-date Ciddor formula can be used, due to the fact that alignment/guiding removes the effect of dispersion in zeroth order. The simplified formula only depends on pressure and temperature. The effect of relative humidity and $\mathrm{CO}_{2}$ concentration is very small. Furthermore, the differential refraction follows very simple scaling rules, i.e. it scales linearly with pressure and the inverse of temperature (in Kelvins).

For short exposures, the optimal strategy is, as expected, still to orient the slits with the parallactic angle. On the other hand, for longer exposures, this is not always the right strategy. There are two effects to consider, the increasing differential refraction and the changing angle between the slits and the parallactic angle. Depending on the configuration, these effects can work against each other, thus resulting in a long, relatively stable observation that is never optimal or these effects 


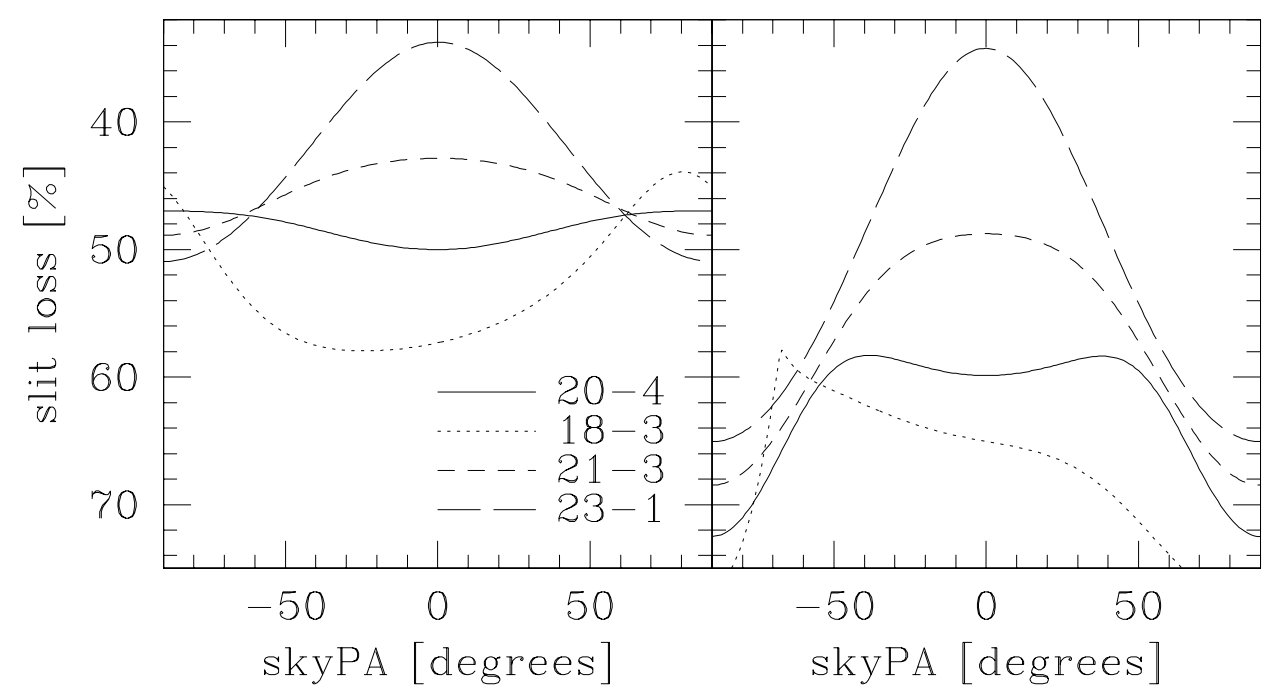

Fig. 5. Average slit loss at $4500 \AA$ at different slit orientations. I used our sample observation of the Lockman Hole using Keck (see Sect. 1 for details - left panel) and a southern field (CDFS, -27:48:30 declination - right panel). I show the effect of slit sky position angle at different hour angle ranges. For sky position angle I use the (unusual) DEIMOS definition, where 90 degree is E-W, 0 degree is $\mathrm{N}-\mathrm{S}$.

can amplify each other, thus resulting in an optimal short observation that deteriorates very fast.

Alignment/guiding is crucial. One has to select the effective wavelength of these to maximize the science output. It is worthwhile to spend a few extra minutes every few hours using some standard filters instead of using no filters at all, especially with alignment stars with unknown spectral types. This latter approach runs the risk of using very blue stars for alignment, thus the alignment will only be optimal in the blue, where one may not be observing.

Naturally, in the long run, the use of atmospheric dispersion correctors should be considered. As I have shown, these can improve the throughput by as much as a factor of two for instruments operating in the blue. The cost of these from an observational point of view is small (few photons are lost in the extra optical elements) as only two very weak prisms are sufficient in most cases to produce a "tunable" prism to compensate for differential refraction in the first order. This can solve the problem of differential refraction, but does not eliminate the slit loss completely. Finite slit widths will always "cut" the object signal. As the seeing can be wavelength dependent (this effect was completely ignored in this paper), so can the slit loss. Thus, accurate spectrophotometry still requires very wide slits, and consequently, very low spectral resolution and a significantly degraded signal to noise ratio.

Finally, I provide a web-based service to the community and release the software developed.

Acknowledgements. Part of this work was supported by the German DLR project number 50 OX0001.

\section{References}

Ciddor, P. E. 1996, Appl. Opt., 35, 1566

Cohen, J. G., \& Cromer, J. 1988, PASP, 100, 1582

Donnelly, R. H., Brodie, J. P., Bixler, J. V., \& Hailey, C. J. 1989, PASP, 101,1046

Cuby, J. G., Bottini, D., \& Picat, J. P. 1996, Proc. SPIE, 3355, 36

ESO, 2005, VIMOS User Manual, Doc. No. VLT-MAN-ESO-146103509,16

Filippenko, A. V. 1982, PASP, 94, 715

Saunders, P. 1990, World Ocean Circulation Experiment Newsletter, 10,10 


\section{Online Material}




\section{Appendix A: Atmospheric dispersion}

Atmospheric dispersion (i.e. the apparent displacement of object), $R$, is defined as

$R=z_{\mathrm{t}}-z_{\mathrm{a}}$,

where $z_{\mathrm{t}}$ and $z_{\mathrm{a}}$ are the true and apparent zenith distances, respectively.

Assuming that the index of refraction depends only on height, using Snell's law, we can write that

$n(h) \sin (z(h)) \equiv$ const.

where $h$ is the height, $z(h)$ is the apparent zenith distance at height $h$.

$n \sin z_{\mathrm{a}}=\sin z_{\mathrm{t}}$.

Thus, the apparent zenith distance at the telescope only depends on the index of refraction at the observatory. Assuming that $R$ is small, i.e. $\sin R \approx R$ and $\cos R \approx 1$, we can write

$R \approx(n-1) \tan z_{\mathrm{a}}$.

As no telescope points accurately enough (i.e. with less than the 0.1 arcsec accuracy required by slit based spectroscopy), all observations start with an "alignment". This step guarantees that all objects are centered on the slit at a particular wavelength, $\lambda_{0}$ (determined by the filter used for the alignment). During the exposure, the guiding subsystem and periodic realignments maintain this condition. As the guider typically does not operate at the same wavelength, special care is required to compensate for atmospheric refraction in the guider system. Even with an ideal guider, the mask gets misaligned due to other effects, e.g. the open loop instrument rotator (especially on modern, altitude-azimuth mounted telescopes). Thus, a periodic realignment is mandatory. For simplicity, I will assume that the telescope is equiped with an ideal alignment and guiding system, thus the objects are always centered on the slit at wavelength $\lambda_{0}$. In Sect. 6 I discuss why this cannot hold for wide field-ofview instruments.

As a consequence, the absolute magnitude of atmospheric refraction is irrelevant, since alignment/guiding automatically compensates for it. The relevant quantity, the differential refraction (as a function of wavelength, relative to the alignment/guiding effective wavelength, $\lambda_{0}$ ) in radians is:

$\Delta R(\lambda) \equiv R(\lambda)-R\left(\lambda_{0}\right)=\left(n(\lambda)-n_{0}\right) \tan z_{\mathrm{a}}$.

The calculation of the refractive index is a crucial part of our calculation. Unfortunately, there are still old formulas in use, most notably the Cauchy formula and the old and new Edlén formulas that are at least 50 years old. These formulas are known to be inaccurate, but they still are seen in the literature and astronomical applications. The current best formula is the Ciddor (1996) formula, presented below.

The index of refraction, $n_{\mathrm{as}}$ of standard air, i.e. dry air at $15{ }^{\circ} \mathrm{C}$, using the International Temperature Scale of 1990 (Saunders 1990), $101325 \mathrm{~Pa}$ pressure and 450 ppm (part per million) $\mathrm{CO}_{2}$ concentration, is

$10^{8}\left(n_{\mathrm{as}}-1\right)=\frac{5792105 \mu \mathrm{m}^{-2}}{238.0185 \mu \mathrm{m}^{-2}-\sigma^{2}}+\frac{167917 \mu \mathrm{m}^{-2}}{57.362 \mu \mathrm{m}^{-2}-\sigma^{2}}$, (A where $\sigma$ is the wave number (reciprocal of the vacuum wavelength) in inverse micrometers. In the range of $3500 \AA . .24000 \AA, 10^{8}\left(n_{\mathrm{as}}-1\right)$ is in the range of $28612 \ldots 27289$.

If the $\mathrm{CO}_{2}$ concentration is $x_{\mathrm{c}} \mathrm{ppm}$ instead of $450 \mathrm{ppm}$, the index of refraction, $n_{\mathrm{axs}}$, is

$n_{\mathrm{axs}}-1=\left(n_{\mathrm{as}}-1\right)\left(1+0.534 \times 10^{-6}\left(x_{\mathrm{c}}-450\right)\right)$.

This formula is accurate to $10^{-8}$ for the refractive index up to $600 \mathrm{ppm} \mathrm{CO} 2$ concentrations in the range of $360-2500 \mathrm{~nm}$. In this range, the effect of $\mathrm{CO}_{2}$ variation on $n_{\mathrm{axs}}$ is on the order of $10^{-7}$.

For water vapor at the "standard conditions", i.e. at $20^{\circ} \mathrm{C}$ and $1333 \mathrm{~Pa}$, the index of refraction, $n_{\mathrm{ws}}$, is

$$
\begin{aligned}
10^{8}\left(n_{\mathrm{ws}}-1\right) & =1.022\left(295.235 \mu \mathrm{m}^{-2}+2.6422 \mu \mathrm{m}^{-2} \sigma^{2}\right. \\
& \left.-0.032380 \mu \mathrm{m}^{-4} \sigma^{4}+0.004028 \mu \mathrm{m}^{-6} \sigma^{6}\right) .
\end{aligned}
$$

The formula is accurate to $2 \times 10^{-7}$ in the range of $350-1200 \mathrm{~nm}$. In the optical/near-IR range $\sigma$ is between 0.5 and 3 . Thus, the value of $10^{8}\left(n_{\mathrm{ws}}-1\right)$ is $324 \ldots 302$ in this wavelength range.

The saturation vapor pressure of water vapor, $p_{\mathrm{vs}}$, at temperature $T$ (in Kelvins), over liquid water is

$$
\begin{aligned}
p_{\mathrm{vs}}= & \exp \left(1.2378847 \times 10^{-5} \mathrm{~K}^{-2} T^{2}\right. \\
& -1.9121316 \times 10^{-2} \mathrm{~K}^{-1} T \\
& +33.93711047-6343.1645 \mathrm{~K} / T) .
\end{aligned}
$$

Considering a temperature range of $-20^{\circ} \mathrm{C} \ldots 40^{\circ} \mathrm{C}$, the saturated vapor pressure is $0.1 \ldots 7.4 \mathrm{kPa}$.

The enhancement factor of water vapor in air is

$f=1.00062+3.14 \times 10^{-8} \mathrm{~Pa}^{-1} p+5.6 \times 10^{-7}{ }^{\circ} \mathrm{C}^{-2} t^{2}$,

where $p$ is the pressure and $t=T-273.15 \mathrm{~K}$. The deviation of $f$ from 1 is at most $4 \times 10^{-4}$.

The molar fraction of water vapor in moist air is

$x_{\mathrm{w}}=\frac{f h p_{\mathrm{vs}}}{p}$,

where $h$ is the fractional humidity (between 0 and 1 ). The range of $x_{\mathrm{w}}$ is $0 \ldots 0.25$, but the high value (0.25) assumes unrealistic conditions, i.e. $40{ }^{\circ} \mathrm{C}$ temperature, $100 \%$ humidity and $30 \mathrm{kPa}$ ambient pressure. In most cases, accepting a range of $0 . \ldots 0.05$ $\left(20^{\circ} \mathrm{C}, 60 \mathrm{kPa}\right)$ is more realistic.

The compressibility of the moist air, $Z$ is

$$
\begin{aligned}
Z & =1-\frac{p}{T}\left[1.58123 \times 10^{-6} \mathrm{~K} \mathrm{~Pa}^{-1}\right. \\
& -2.9331 \times 10^{-8} \mathrm{~Pa}^{-1} t \\
& +1.1043 \times 10^{-10} \mathrm{~K}^{-1} \mathrm{~Pa}^{-1} t^{2} \\
& +\left(5.707 \times 10^{-6} \mathrm{KPa}^{-1}-2.051 \times 10^{-8} \mathrm{~Pa}^{-1} t\right) x_{w} \\
& \left.+\left(1.9898 \times 10^{-4} \mathrm{KPa}^{-1}-2.376 \times 10^{-6} \mathrm{~Pa}^{-1} t\right) x_{w}^{2}\right] \\
& +\left(\frac{p}{t}\right)^{2}\left(1.83 \times 10^{-11} \mathrm{~K}^{2} \mathrm{~Pa}^{-2}\right. \\
& \left.-0.765 \times 10^{-8} \mathrm{~K}^{2} \mathrm{~Pa}^{-2} x_{w}^{2}\right),
\end{aligned}
$$


where $p$ is pressure (in Pascals), $T$ is temperature (in Kelvins) and $t=T-273.15 \mathrm{~K}$. Considering realistic pressures $(30 \ldots 100 \mathrm{kPa})$, temperatures $\left(-20 \ldots 40^{\circ} \mathrm{C}\right)$ and water vapor molar fractions $\left(x_{\mathrm{w}}<0.1\right)$, the compressibility is very close to one: $|Z-1|<2 \times 10^{-3}$ (using a very conservative upper limit estimate).

At standard conditions ( $p=101325 \mathrm{~Pa}, t=15^{\circ} \mathrm{C}$, dry air), the standard compressibility is

$Z_{0} \approx 0.9995922115$.

At the saturated water vapor conditions, i.e. $p=1333 \mathrm{~Pa}$ and $t=20^{\circ} \mathrm{C}$, the compressibility is

$Z_{1} \approx 0.9999952769$.

The molar mass of air is

$M_{\mathrm{a}}=10^{-3}\left(28.9635+12.011 \times 10^{-6}\left(x_{\mathrm{c}}-400\right)\right)$

in $\mathrm{kg} / \mathrm{mol}$ units $\left(x_{\mathrm{c}}\right.$ is the $\mathrm{CO}_{2}$ concentration in $\mathrm{ppm}$, as used above).

The density of air, $\rho$ (in $\mathrm{kg} / \mathrm{m}^{3}$ units) is

$\rho=\frac{p M_{\mathrm{a}}}{Z R T}\left(1-x_{\mathrm{w}}\left(1-\frac{M_{\mathrm{w}}}{M_{\mathrm{a}}}\right)\right)$,

where $R=8.314510 \mathrm{~J} \mathrm{~mol}^{-1} \mathrm{~K}^{-1}$, the gas constant, $M_{\mathrm{w}}=$ $0.018015 \mathrm{~kg} / \mathrm{mol}$, the molar mass of water vapor.

At standard conditions $\left(p_{0}=101325 \mathrm{~Pa}\right.$ and $\left.t_{0}=15^{\circ} \mathrm{C}\right)$ the density of dry air $\left(x_{\mathrm{w}}=0\right)$ only depends on the $\mathrm{CO}_{2}$ concentration

$$
\begin{aligned}
\rho_{\mathrm{axs}}= & \frac{p_{0} M_{\mathrm{a}}}{Z_{0} R T_{0}}=\frac{28.9635 \times 10^{-3} 3 p_{0}}{Z_{0} R T_{0}} \\
& \times\left(1+\frac{12.011}{28.9635} \times \frac{x_{c}-400}{10^{6}}\right) .
\end{aligned}
$$

At water vapor standard conditions $\left(p_{1}=1333 \mathrm{~Pa}, t_{1}=20^{\circ} \mathrm{C}\right.$, $\left.w_{\mathrm{s}}=1\right)$, the saturated water vapor density is

$\rho_{\mathrm{ws}}=\frac{p_{1} M_{\mathrm{w}}}{Z_{1} R T_{1}} \approx 0.00985235$.

For actual conditions, the air density of the air component is

$\rho_{\mathrm{a}}=\frac{p M_{\mathrm{a}}}{Z R T}\left(1-x_{\mathrm{w}}\right)$

and

$\frac{\rho_{\mathrm{a}}}{\rho_{\mathrm{axs}}}=\frac{Z_{0}}{Z} \frac{p}{p_{0}} \frac{T_{0}}{T}\left(1-x_{\mathrm{w}}\right)$.

The water vapor component is

$\rho_{\mathrm{v}}=\frac{p M_{\mathrm{w}} x_{\mathrm{w}}}{Z R T}$

and

$\frac{\rho_{\mathrm{v}}}{\rho_{\mathrm{ws}}}=\frac{p}{p_{1}} \frac{T_{1}}{T} \frac{Z_{1}}{Z} x_{\mathrm{w}}=\frac{T_{1}}{T} \frac{Z_{1}}{Z} \frac{p_{\mathrm{vs}}}{p_{1}} f h$.

Considering that $Z$ and $f$ are very close to one, assuming realistic ranges for the parameters, we can place a very conservative
Table A.1. The effect of different environmental parameters on the differential refraction. The effects of changes in the atmospheric parameters on the differential refraction are shown using our observations of the Lockman hole with Keck. As a reference point, $2.5^{\circ} \mathrm{C}$ temperature, $61.5 \mathrm{kPa}$ ambient pressure, $40 \%$ relative humidity and $450 \mathrm{ppm} \mathrm{CO}_{2}$ concentration is used. The effect of changing one of these four parameters (while keeping the other three at the nominal value) is shown below. Only the differential refraction for $3500 \AA$ is shown, $\Delta R(3500 \AA)$ in arc seconds. The hour angle is $18 \mathrm{~h}$.

\begin{tabular}{lc}
\hline \hline Parameter change & $\Delta R(3500 \AA)$ \\
\hline nominal & 4.53 \\
$t=-10{ }^{\circ} \mathrm{C}$ & 4.75 \\
$t=20^{\circ} \mathrm{C}$ & 4.27 \\
$p=30 \mathrm{kPa}$ & 2.21 \\
$p=100 \mathrm{kPa}$ & 7.37 \\
$\mathrm{RH}=0 \%$ & 4.53 \\
$\mathrm{RH}=90 \%$ & 4.54 \\
$x_{\mathrm{c}}=300 \mathrm{ppm}$ & 4.53 \\
$x_{\mathrm{c}}=500 \mathrm{ppm}$ & 4.53 \\
\hline
\end{tabular}

upper limit on the vapor density to standard saturated water vapor density ratio: $\rho_{\mathrm{v}} / \rho_{\mathrm{ws}}<6.5$.

Finally, the refractive index is

$n-1=\frac{\rho_{\mathrm{a}}}{\rho_{\mathrm{axs}}}\left(n_{\mathrm{axs}}-1\right)+\frac{\rho_{v}}{\rho_{\mathrm{ws}}}\left(n_{\mathrm{ws}}-1\right)$.

As I have shown above, $n_{\mathrm{ws}}$ varies by at most $2 \times 10^{-7}$ as a function of wavelength in the optical/near-IR range and $\rho_{v} / \rho_{\mathrm{ws}}<$ 6.5. Thus, the second, water-related term can change by at most $1.3 \times 10^{-6}$, thus the differential refraction can change by at most this much. This limits the effect of water in the atmosphere to 0.3 arcsec in the most extreme case: alignment/guiding in the $K$-band, observing in the UV, close to $100 \%$ humidity. In a realistic case, the effect is much smaller so the second term can be ignored most of the time. The first term in the equation does depend on humidity through the air density, but in realistic cases the only water-related term, $1-x_{\mathrm{w}}$ varies by a few percent, thus the effect is very small.

As I have shown, the variation in $n_{\text {axs }}$ introduced by $\mathrm{CO}_{2}$ concentration variation is less than $10^{-7}$. Thus, the differential refraction variation introduced is 0.03 arcsec or less. For most applications, this is negligible.

If we are only interested in differential refraction and are considering these approximations, we can write a much simpler formula that is sufficiently accurate for many applications:

$\Delta R(\lambda) \approx \frac{p T_{0}}{p_{0} T}\left(n_{\mathrm{as}}(\lambda)-n_{\mathrm{as}}\left(\lambda_{0}\right)\right) \tan z_{\mathrm{a}}$.

In Table A. 1 the importance of atmospheric condition variations is shown. The effect of relative humidity and $\mathrm{CO}_{2}$ concentration is negligible in most observations. 